\title{
Modified Adaptive Notch Filter Based on Neural Network for Flexible Dynamic Control
}

\author{
Ali Elmelhi
}

\begin{abstract}
The structure frequency bending modes are the limiting factor to achieve a stability margins and performance of the flight vehicle Autopilots. This is due to a lightly damping structure of these bending frequency modes. The structure engineer could evaluate these frequencies at different design operating points according to the locations of rate gyro sensors on the vehicle beam. Previously, the classical adaptive notch filter can be designed in order to overcome the instability which may occur due to uncertainty of these modes. In this paper, the modified adaptive notch filter is presented in which the classical integral of frequency tracking system in classical adaptive notch filter is replaced by a Radial Basis Function Neural Network for integrator. This modification attempts to enlarge the tracking range of the classical adaptive filter so that the frequency modes with large uncertain values can be gain stabilized. Finally, to demonstrate the objective of this study work, the Simulation results for a typical flexible dynamic model are included at the end of this paper.
\end{abstract}

Index Terms-Flexible model dynamics, high frequency modes, adaptive filter, structure adaptive filter, neural network.

\section{INTRODUCTION}

Damping is the dissipation of energy in a system such that the amplitude and duration of response of the system are reduced. When excited, a lightly damped structure will exhibit large responses at its resonant or natural frequencies. Launch vehicles frequently experience a reduced stability margin due to these excited resonance frequencies called bending frequency modes which has always presented a stability problem because the sensors utilized for controlling the vehicle also respond to the structural bending of the vehicle experiences during flight. If the oscillations of the vehicle due to structural bending modes are of sufficient magnitude, and if the phase of these oscillatory signals is such that the resulting control engine motion reinforces or Previously, the influence of the bending frequency modes on the Autopilot stability margins have been early studied in Space Technical lab report in [1]. The dynamics with controls analysis of flexible vehicles in terms of the natural modes has been studied by [2], where the applied method has been proven very satisfactory for accurately describing not only the mode shape, but its slope and mode shears has been presented.

Further, the influence of these modes on the stability of the flight vehicle have been solved by using of various filtering

Manuscript received October 6, 2013; revised November 19, 2013. This work was supported in part by the University of Tripoli - Libya. The Paper title is (Modified Adaptive Notch Filter Based on Neural Network for Flexible Dynamic Control).

Ali Elmelhi is with University of Tripoli, Faculty of Engineering, Tripoli-Libya (e-mail: ali_elmulhi@yahoo.com) techniques [3], [4], and their effects on the system tracking performance of a typical launch vehicle have been studied and analyzed in [5].

In order to achieve the system stabilization in case of uncertain structure frequency modes, an alternative design approach by using of an adaptive tracking filter was presented which can track the frequency of the predominant oscillatory component of its input signal automatically and adjust its shaping charterstics as a function of that frequency [6], [7]. Where a stabilization of the closed feedback control systems to these undesirable structural bending mode signals can be accomplished by either gain-stabilization or phase-stabilization techniques.

The RBFNN methodology was introduced in 1971 by Rolland Hardy [8]. RBF emerged as a variant of artificial neural networks in late 80's and the first of its application in neural networks have been studied in [9]. Many RBF papers and references can be found in the recent Neurocomputing special issues on RBF networks [10]. Furthermore, the RBF has been widely used in multivariate interpolation, neural network, time-series prediction, control of nonlinear systems, mesh-free approximation and target tracking in video data [11], [12].

Recently, an adaptive integral dynamic surface control approach based on fully tuned RBF neural network has been presented for a general class of strict-feedback nonlinear systems, which may possess a wide class of uncertainties that are not linearly parameterized and do not have any prior knowledge of the bounding functions [13]. And the uncertainty can be approximated online, and a systematic framework for adaptive controller design is given by dynamic surface control. The control algorithm has two outstanding features, namely, the neural network regulates the weights, width and center of Gaussian function simultaneously, which ensures the control system has perfect ability of restraining different unknown uncertainties and the integral term of tracking error introduced in the control law can eliminate the static error of the closed loop system effectively.

Motivated by the aforementioned issues, in this study, the RBFNN control scheme is incorporating with the conventional structure notch filter dynamics through a frequency tracking system to get a modified filter structure called RBFNN adaptive notch filter.

The key point in this design concept is to replace the classical integrator in frequency tracking system by RBFNN for integration, where the RBFNN dynamics described by the centers, widths of the Gaussian function and output weighting parameters are represent the classical integrator. It is anticipated that, this modification will take the advantages of the classical adaptive tracking notch filter with more 
powerful capability of learning and adaptability to track the frequency modes with a wide range of uncertainties. In order to validate the effectiveness and superiority of this modified design, the comparison with classical adaptive notch filter is carried out where only one frequency mode is considered.

The total task of this work was subdivided into the following steps; the rigid body and flexible dynamic models are outlined respectively in Section II and Section III. The frequency mode stabilization was given in Section IV.

Computer simulation results are shown in Section V, and finally the conclusion with recommendations are included in Section VI.

\section{RIGID BODY DYNAMIC MODEL}

In case of a rigid body dynamics, let the motion of the considered symmetrical launch vehicle is only restricted in one plane $x_{b} y_{b}$ as shown in Fig. 1. And due to weak interaction between motions around the three axes $x_{b} y_{b} z_{b}$ is often considered, since the control system keeps angular velocities and angles small. Consequently it is preferred to assume that the launch vehicle is completely rigid where the effect of flexibility due to high frequency bending modes is neglected. In this paper, the launch vehicle geometry given in [14] is considered here. Where the force and moment equations in pitch plane are reduced into

$$
\begin{gathered}
\sum F_{z}=-m_{T} g \theta \cos \left(\theta_{0}\right)+T_{c} \delta-L_{\alpha} \\
\sum M_{z}=T_{c} l_{c} \delta+L_{\alpha} l_{\alpha} \alpha
\end{gathered}
$$

where $m_{T}$ is the total mass of the considered vehicle $(\mathrm{kg})$, $\theta$; pitch angle (deg), $\theta_{0}$; the initial value of pitch angle before launching ( $\operatorname{deg}$ ), $L_{\alpha}$; aerodynamic load per unit angle of attack $\alpha, l_{\alpha}$; distance from mass center of vehicle $c_{m}$ to the center of pressure $c_{p}, T_{c}$; effective thrust (Newton), $\delta$; elevator control deflection ( $\operatorname{deg}$ ), $l_{c}$; the distance between $c_{m}$ to the engine swivel point,and $\theta_{0}$ is the initial value of pitch angle and $g$ is the earth gravitation force meter. $\sec ^{2}$, the total moment in (2) can be written in the following form [15]:

$$
I_{y y} \ddot{\theta}=T_{c} l_{c} \delta+L_{\alpha} l_{\alpha} \alpha
$$

where $I_{y y}$; moment of inertia in $y$ axes $k g$. meter $^{2}, \alpha$; is the angle of attack deg. This moment equation is considered due to its direct relation to the stabilization of the launch vehicle in the longitudinal motion. And from (3), the rigid body dynamic model can be expressed by the following simplified transfer function [14]:

$$
\frac{\theta}{\delta}=\frac{k_{c}}{s^{2}+k_{\alpha}}
$$

where $k_{c}=\frac{T_{c} l_{c}}{I_{y y}}, \quad k_{\alpha}=\frac{L_{\alpha} l_{\alpha}}{I_{y y}}$

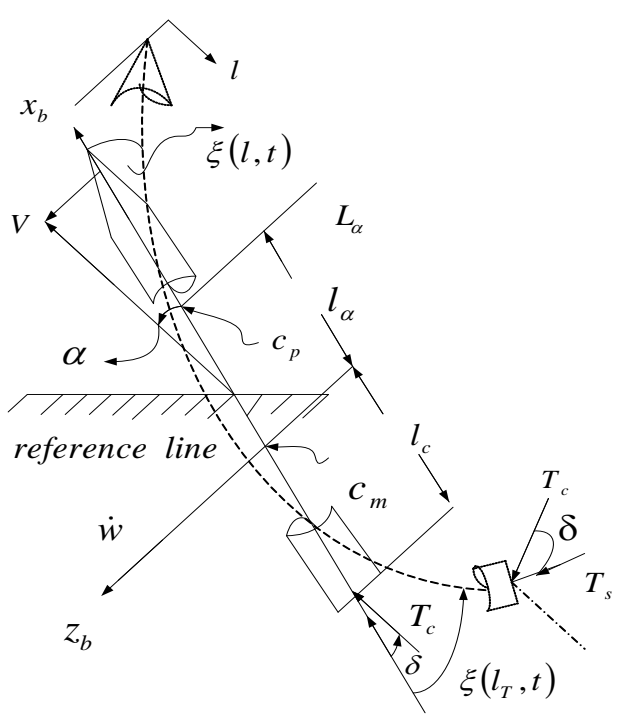

Fig. 1. Geometrical diagram for flexible vehicle dynamic model

\section{Elastic DyNAMiC MODEL}

The structure of the launch vehicle usually has a small rigidity. Thus under the effect of external force, this type of structure can produce a deformation and bending vibrations (elastic vibration). Generally, the elastic vibration has a longitudinal, torsion and horizontal vibrations. Where, the longitudinal vibrations are perpendicular to the elastic shift of the longitudinal axis of the flight vehicle. This type of effect is usually very small but as far as the carrying system of the propellant of the large liquid launch vehicle is concerned, its effect is very large. As a result, the effect of the coupled longitudinal vibrations should be considered during attitude control motion and stability analysis. And due to assumption of the symmetry, the coupling of the torsion and horizontal vibrations with longitudinal motion is negligible. The dashed axis shown in Fig. 1 illustrates the longitudinal axis after deformation due to effect of elastic vibrations. Where $\dot{w}$ denotes to the acceleration in $z$-axes (meter $/ \sec ^{2}$ ), $V$ is the vehicle relative velocity (meter/ $\mathrm{sec}$ ).

And the amount of deflection due to effect of the elastic vibrations on the longitudinal axis is represented by the following Fourier series function [14], [15]:

$$
\xi(l, t)=\sum_{i=1}^{n} q_{i}(t) W_{i}(l) \quad i=1,2, \ldots \ldots n
$$

where $i$ is the number of the considered modes which are usually not exceed five modes and $l$ is the length of the vehicle longitudinal axis. The function $W_{i}(x)$ shows the relative horizontal shift relation of each point on the longitudinal axes of flight vehicle called ith natural vibration function, $q_{i}$ can decide the elastic vibration amplitude after determination of elastic vibration function which always satisfies the following differential equation:

$$
\ddot{q}_{i}+2 \varsigma_{i} \omega_{i} \dot{q}_{i}+\omega_{i}^{2} q_{i}=-\frac{Q_{i}}{M_{i}}
$$

where for each ith mode, $\omega_{i}$ denotes to the natural frequency mode, $\varsigma_{i}$ is the damping coefficient, $Q_{i}$ is the 
generalized force and $M_{i}$ represents the generalized mass. If the excitation of the ith mode is considered only due to thrust and engine deflection forces, then the previous equation can be rewritten in the following form: [14]

$$
\ddot{q}_{i}+2 \varsigma_{i} \omega_{i} \dot{q}_{i}+\omega_{i}^{2} q_{i}=-\frac{1}{M_{i}}\left(m_{r} l_{r} \ddot{\delta}+T_{c} \delta\right) W_{i}
$$

where, $m_{r}$ denotes to the mass of swinging portion of a single engine ( $\mathrm{kg}$ ), and $l_{r}$ is the distance from the vehicle center of mass to swing axis (meter). And the point location of thrust and engine deflection forces is at the vehicle engine swivel point $l=L_{T}$. However, the bending modes are normalized at $l=L_{T}$ such that $W_{i}\left(L_{T}\right)=1$. And by taking the Laplace transform operation of (7), the elastic model dynamics can be represented by the following transfer function:

$$
\frac{q_{i}}{\delta}=\frac{\left(\frac{-m_{r} l_{r}}{M_{i}}\right)\left(s^{2}+\left(\frac{T_{c}}{m_{r} l_{r}}\right)\right)}{s^{2}+2 \varsigma_{i} \omega_{i} s+\omega_{i}^{2}}
$$

If the effect of the frequency bending modes appears only through the output measurement rate gyro sensor as shown in Fig. 2 then the feedback signal $\theta_{f}$ is given by the following equation:

$$
\theta_{f}=s K_{R}\left(\theta+\sigma_{G}^{i} q_{i}\right)
$$

where, the rate gyro dynamics is modeled by $s K_{R}$ with $K_{R}$ denotes to the rate gyro gain, $\sigma_{G}^{i}$ is the negative slope of the ith bending mode in pitch plane where the gyro is located [14].

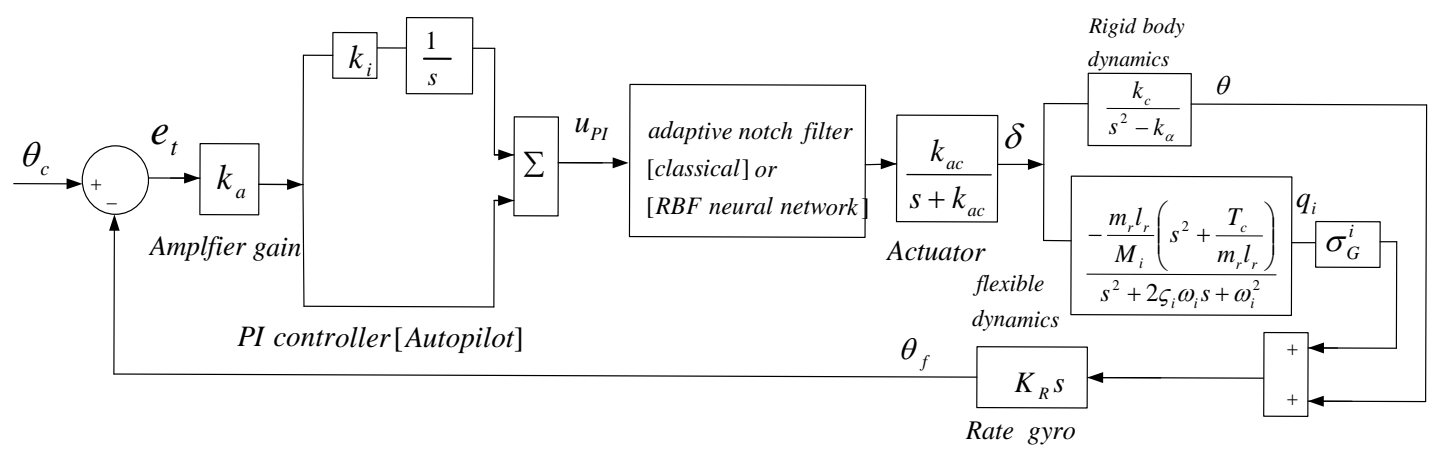

Fig. 2. Closed loop system with adaptive notch filter

\section{FrequenCy Mode StabiLIZATION}

In this section, the system is compensated by using of PI controller with the lack of frequency bending modes [rigid body dynamic model]. Next, an additional compensation is inserted in the forward loop by using of classical or RBFNN adaptive notch filters in order to discard the effects of these modes when the flexibility of vehicle dynamic model is taken into account. This can be explained in more details as follows.

\section{A. Rigid Body Compensation}

In order to stabilize the rigid dynamic model of the considered vehicle, the simple classical PI controller is used for Autopilot design. Where, the controller gains are adjusted so that an acceptable overshoot and zero steady state error can be satisfied. This type of controller can be described by the following simple generic model: [14]

$$
u_{P I}=k_{a}\left(e(t)+k_{i} \int_{0}^{\infty} e(t) d t\right)
$$

where $k_{\alpha}$ denotes to the amplifier gain and $k_{i}$ represents the integral gain. And the error signal $e(t)$ is given by the following difference equation:

$$
e(t)=\theta_{c}(t)-\theta_{f}(t)
$$

\section{B. Classical Adaptive Notch Filter}

When the flexible dynamics are considered as shown by
Fig. 2, the stability of this system is examined under the influence of frequency elastic bending modes $\omega_{i}$. In this situation, the classical PI controller design is supported by cascade dynamics of the structural notch filter for gain stabilization with arbitrary complex poles and zeros described by the following generic second order transfer function [16]:

$$
G_{F}=\frac{\left(\frac{s^{2}}{\omega_{z}^{2}}+\frac{2 \varsigma_{z} s}{\omega_{z}}+1\right)}{\left(\frac{s^{2}}{\omega_{p}^{2}}+\frac{2 \varsigma_{p} s}{\omega_{p}}+1\right)}
$$

where the notch filter transfer function $G_{N F}(s)$ can be extracted by assuming that, the natural frequencies $\omega_{p}=\omega_{z}$ and damping ratio $\varsigma_{z}=0$ to get

$$
G_{N F}=\frac{\left(\frac{s^{2}}{\omega_{p}^{2}}\right)}{\left(\frac{s^{2}}{\omega_{p}^{2}}+\frac{2 \varsigma_{p} s}{\omega_{p}}+1\right)}
$$

The sharpness of this filter is mainly depends on the damping ratio $\varsigma_{p}$. And the gain stabilization is considered here which provides an attenuation of the control loop gain at the frequency mode to ensure the stability regardless of the phase uncertainties. 


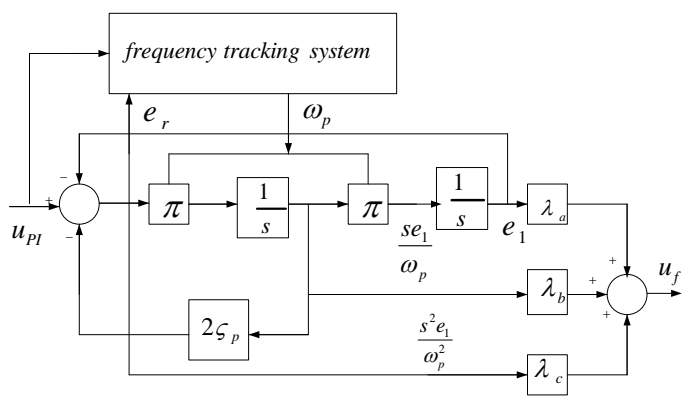

Fig. 3. General adaptive notch filter block diagram

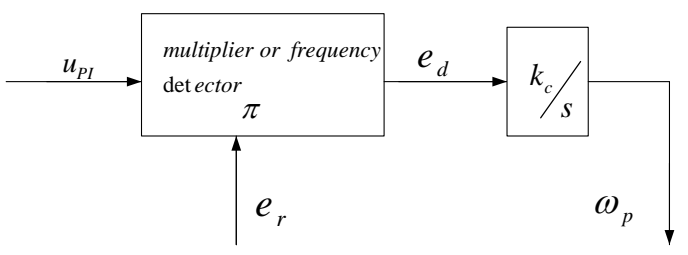

Fig. 4. Classical frequency tracking system

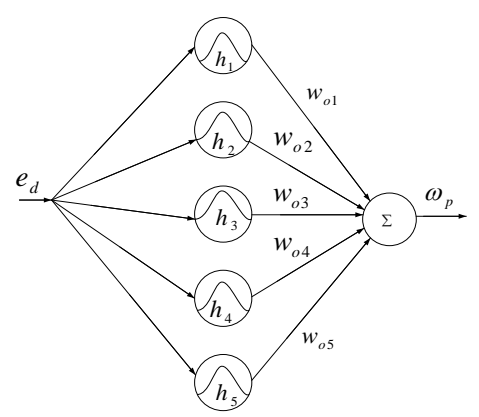

Fig. 5. RBFNN structure for integrator in frequency tracking system

Due to variations in the structural mode parameters (high frequency uncertainties), the classical notch filter represented by the previous equation fails to achieve the gain stabilization of the uncertain modes. In order to satisfy the tracking of these frequency modes, the classical adaptive tracking filter is applied here. Fig. 3 shows the block diagram for the tunable adaptive second order notch filter given by (12) with $\left(\omega_{p}=\omega_{z}\right)$ and $\left(\lambda_{a}=\lambda_{c}=1, \lambda_{b}=0\right)$. [16]

The frequency tracking system structure is illustrated in Fig. 4, where its output adjusts the corner frequency $\omega_{p}$ of the considered filter to track the input bending frequency elastic mode $\omega_{i}$. This can be obtained by integrating the frequency error signal (phase shift error $e_{d}$ ) between the input and the reference signal of the tunable notch filter. Where, this error can be evaluated by a simple conventional multiplier [detector]. And the integral gain $k_{c}$ is adjusted until the tracking of the frequency mode is achieved.

\section{RBFNN Adaptive Notch Filter}

In this design case, the RBFNN is applied as a phase shift integrator in frequency tracking system. And its training algorithm is formulated based on the concepts given in [17]. Fig. 5 shows the construction of this network. Where, five neurons are considered in the hidden layer and only the error $e_{d}$ is applied through the input layer. The Gaussian transfer function is used as an activation nonlinear function for hidden layer units. And the integration process can be achieved by summing the phase shift error through the output of this network. It should be noted that, the outputs of the selected nonlinear Gaussian functions in the hidden neurons are proportional to the Euclidian norm of the distance between the input $e_{d}$ and the centers of these functions. This nonlinear mapping between the input and hidden layer with linear mapping through the output of this network attempts to enhance the learning rate and avoid the local minimum problem during training process of the weighting and $\mathrm{RBF}$ parameters.

Let the radial basis vector for hidden layer is given by

$$
\left[h_{1}, h_{2}, \ldots \ldots \ldots . ., h_{n}\right]^{T}
$$

where $h_{j}$ represents the Gaussian nonlinear function for the jth neurons which can be described by the following exponential function:

$$
h_{j}=e^{\frac{\left\|e_{d}-c_{j}\right\|^{2}}{2 b_{j}^{2}}} \quad(j=1,2, \ldots \ldots \ldots m)
$$

With $c_{j}$ and $b_{j}$ are respectively the centers and widths parameters of this function which are the key properties to describe the RBF units. And the \|\| denotes to the Euclidean norm.

And the network output can be evaluated according to the following summation formula:

$$
\omega_{p}=\omega_{p}(k-1)+\sum_{j=1}^{5} h_{j} w_{o j}
$$

where $k$ denotes to an iteration number, $w_{o j}$ represents the weighting parameters for the connections between RBF unit $j$ and network output.

The whole parameters of RBFNN are adjusted so that the following cost function is minimized during steady state time of frequency tracking:

$$
J_{d}=0.5 e_{d}^{2}
$$

If this condition is satisfied, then the unity frequency ratio $\left(\omega_{i} / \omega_{p}\right)$ is approximately achieved, which means that, both frequencies are approximately coincided. This result tends to conclude that, the integral term responds to accumulated errors after processing through the nonlinear Gaussian Function and the output weighting parameters.

Based on the gradient descent algorithm, the weight, node center and radial width iterative algorithms are formulated according to this design problem as follows [17].

$$
\begin{gathered}
w_{o j}(k)=w_{o j}(k-1)+\eta e_{d} h_{j} \\
b_{j}(k)=b_{j}(k-1)+\eta \Delta b_{j} \\
c_{j}(k)=c_{j}(k-1)+\eta \Delta c_{j} \\
\Delta b_{j}=e_{d} w_{o j} h_{j} \frac{\left\|e_{d}-c_{j}\right\|^{2}}{b_{j}^{3}} \\
\Delta c_{j}=e_{d} w_{o j} \frac{e_{d}-c_{j}}{b_{j}^{2}}
\end{gathered}
$$




\section{COMPUTER RESUlts}

For a stability analysis of the considered vehicle control system with the coupling of elastic motions, the closed loop system shown in Fig 2 is simulated based on the typical trajectory and structure data for the considered launch vehicle which are written in Table I.

TABLE I: SIMULATION DATA

\begin{tabular}{|c|c|}
\hline Parameter & Value \\
\hline$\omega_{1}$ & $18.9 \mathrm{rad} / \mathrm{sec}$ \\
\hline$T_{c}$ & $341000 \mathrm{pound}$ \\
\hline$l_{r}$ & $2.52 \mathrm{~m}$ \\
\hline$M_{1}$ & $1590 \mathrm{~kg}$ \\
\hline$k_{c}$ & $4.56 \mathrm{sec}^{-1}$ \\
\hline$k_{\alpha}$ & $2.8 \mathrm{sec}^{-1}$ \\
\hline$m_{r}$ & $30.8 \mathrm{~kg}$ \\
\hline$s_{1}$ & 0.08 \\
\hline$K_{R}$ & $0.33 \mathrm{sec}^{-1}$ \\
\hline$\sigma_{G}^{1}$ & 0.004 \\
\hline$k_{a c}$ & 100 \\
\hline
\end{tabular}

And this simulation is done by using of Matlab/Simulink tool for only one designed elastic frequency mode $\left(\omega_{1}=18.9 \mathrm{rad} / \mathrm{sec}\right)$. Initially, the corner frequency for both types of adaptive notch filters is designed with $\omega_{p}=\omega_{1}$. Table II shows the design parameters for PI controller, classical and RBFNN frequency tracking systems.

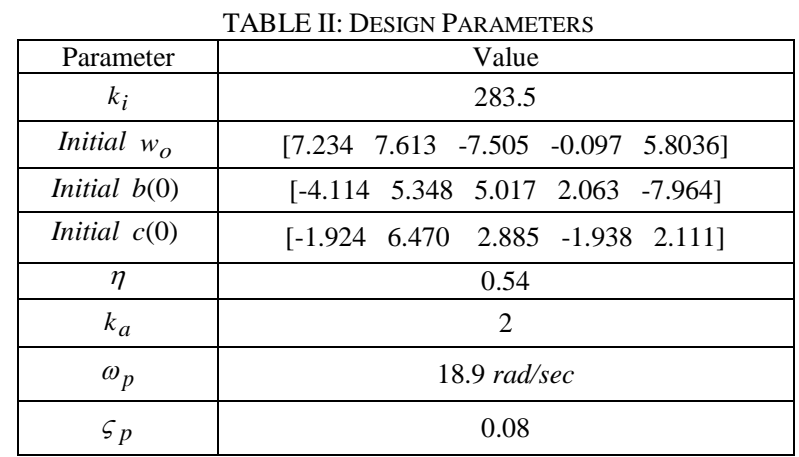

As shown in Fig. 2, the adaptive tracking notch filter is located in the forward loop and it is shaping adaptively in order to compensate the system by simply eliminate the bending frequency terms existed in the feedback measured signal $\theta_{f}$. However, sometimes due to structure variations of the system parameters, the evaluated structure data for the elastic modes are perturbed from their nominal designed values. And in order to carry out the effect of such type of uncertainties, it is assumed that, the considered frequency mode is deviated from its nominal design value $\left(\omega_{1}=18.9 \mathrm{rad} / \mathrm{sec}\right)$ with some uncertain values as shown in Table III.

TABLE III: STEADY STATE CORNER FREQUENCIES FOR ADAPTIVE FILTERS

\begin{tabular}{|c|c|c|}
\hline $\begin{array}{c}\text { First frequency } \\
\text { bending mode } \\
\omega_{1}[\mathbf{r a d} / \mathbf{s e c}]\end{array}$ & $\begin{array}{c}\text { Classical filter } \\
\text { corner frequency } \\
\omega_{p}[\mathbf{r a d} / \mathbf{s e c}]\end{array}$ & $\begin{array}{c}\text { RBFNN filter corner } \\
\text { frequency } \\
\omega_{p}[\mathbf{r a d} / \mathbf{s e c}]\end{array}$ \\
\hline 18.9 & 18.59 & 18.97 \\
\hline 14 & 14.29 & 13.96 \\
\hline 12 & Fails to track & 12.51 \\
\hline 10 & Fails to track & 10.27 \\
\hline 9 & Fails to track & 8.86 \\
\hline
\end{tabular}

It is seen that, the classical adaptive notch filter fails to track the uncertain frequencies which are beyond the designed value at $\omega_{1}=18.9 \mathrm{rad} / \mathrm{sec}$ and the $\mathrm{RBFNN}$ adaptive notch filter keep tracking of these uncertainties.

In order to investigate the effectiveness of the RBFNN adaptive filter, the linear simulation results are carried out under the influence of uncertain mode $\omega_{1}=9 \mathrm{rad} / \mathrm{sec}$, where it is observed that, the RBFNN adaptive filter is succeeded to track this uncertain value as shown in Fig. 6 while the adaptive classical notch filter fails to track this mode as shown in Fig. 7. This also can be assured from the time step responses of the pitch angle as shown in Fig. 8 in which the instability occur in case of adaptive notch filter. Where the solid and dotted lines are respectively related the classical and RBFNN adaptive filters. In addition, the stability analysis under this uncertain frequency mode can be investigated from the Root Locus and Bode diagrams shown in Fig. 9 and Fig. 10, Where, in contrast to the classical design approach, the RBFNN adaptive filter design can reject the excited peaks of the open loop magnitude curve due to this uncertain mode so that the system can be kept stable. Finally, the investigation of stability can be carried out based on the Root Locus diagram, where it is observed that, the whole closed loop poles which are denoted by bold points at certain gains are located in a stable region.

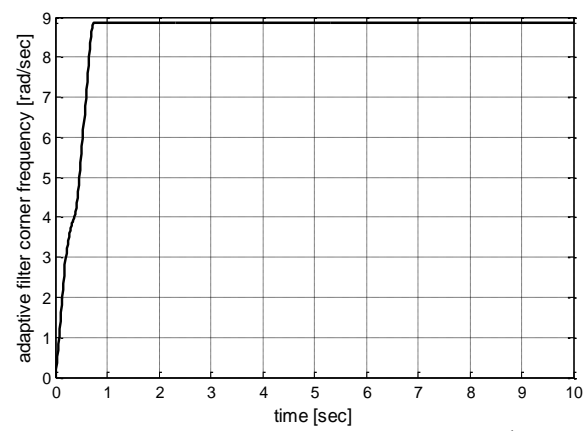

Fig. 6. Frequency tracking for uncertain bending mode $\left(\omega_{1}=9 \mathrm{rad} / \mathrm{sec}\right)$ using RBFNN adaptive notch filter.

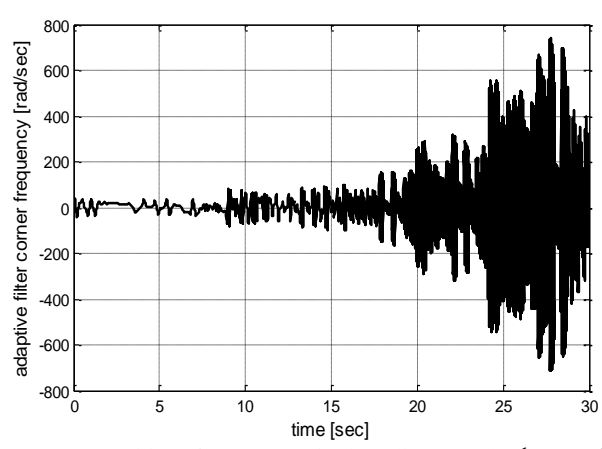

Fig. 7. Frequency tracking for uncertain bending mode $\left(\omega_{1}=9 \mathrm{rad} / \mathrm{sec}\right)$ using classical adaptive notch filter.

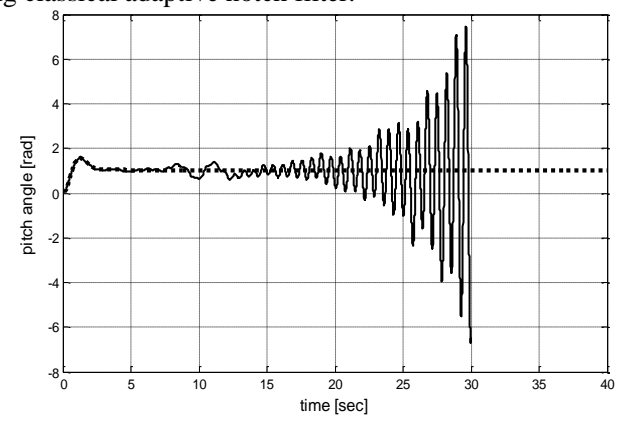

Fig. 8. Step reponses for pitch angle with bending mode $\left(\omega_{1}=\right.$ $9 \mathrm{rad} / \mathrm{sec}$ 

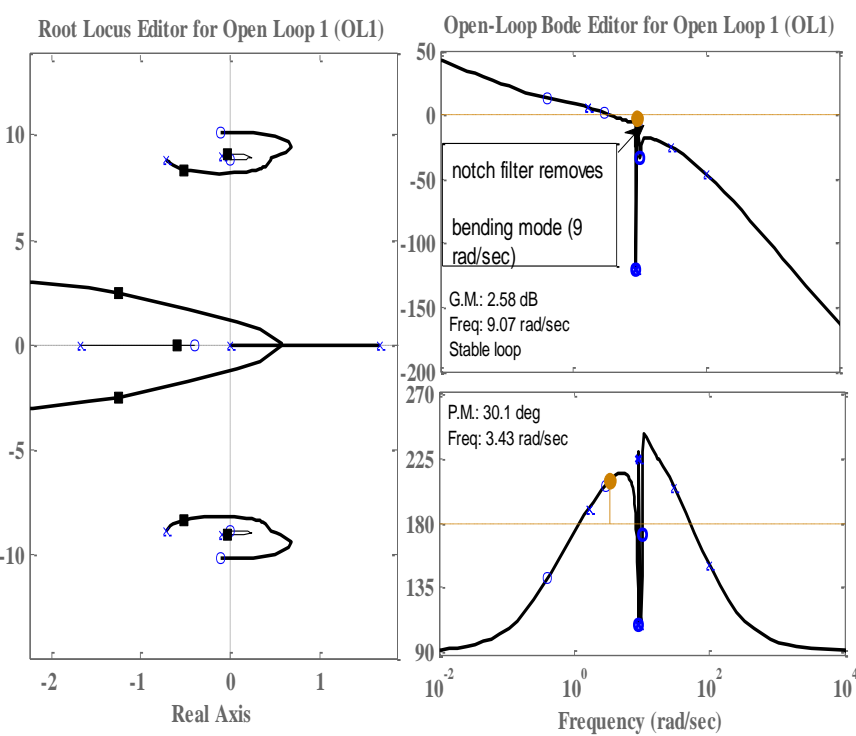

Fig. 9. Root Locus and Bode Diagram for RBFNN adaptive notch filter $\omega_{1}=9 \mathrm{rad} / \mathrm{sec}$.
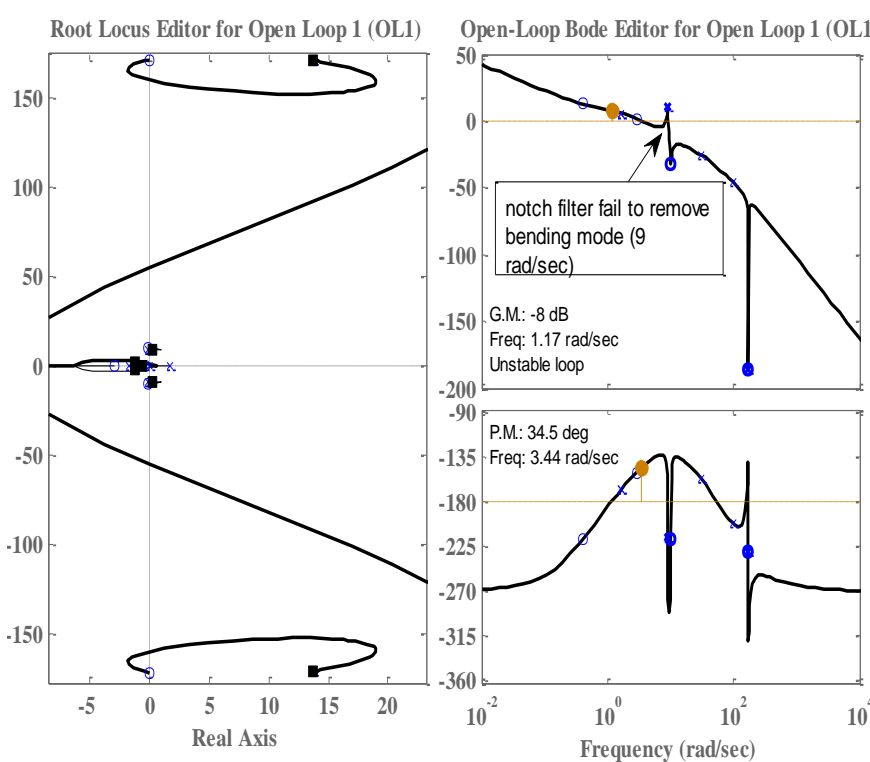

Fig. 10. Root Locus and Bode Diagram for Classical adaptive notch filter $\omega_{1}=9 \mathrm{rad} / \mathrm{sec}$.

\section{CONCLUSION}

The transfer functions of the rigid and flexible body dynamics for the launch vehicle longitudinal motion have been derived. Two design approaches have been taken in order to stabilize the bending modes. The first one is the classical adaptive notch filter, which have been designed so that the uncertain frequency modes can be tracked.

To enlarge the uncertain tracking range, the second design approach based on the Radial Basis Function Neural Network called modified adaptive notch filter was presented. Where, in both design methods, the gain stabilization which provides an attenuation of the control loop gain at these tracked modes is considered. From the computer simulation results, it is concluded that, the modified adaptive notch filter is promised to be a satisfactory means to achieve the tracking of the uncertain frequency modes with a wider range in comparing to the other design approach. From this study and analysis, it is recommended to consider and test the influence of the elastic modes prior in order to avoid the instability which may occur due to inferring of these modes with the Autopilot loop, and to overcome the drawbacks due to flexibility of the vehicle dynamic structure.

\section{REFERENCES}

[1] A. Dite, L. E. Freed, L. F. Lanzer, and B. Lot, Stage I: Autopilot Design Study, Series I, Space Tech. Lab. Rep., May 1958.

[2] V. L. Alley, R. J. Guillotte, and L. D. Hunter, A Method of Determining Model Data of a Non-uniform Beam with Effects of Shear Deformation and Rotary Inertia, NASA Technical Note, No. NASA DID-2930, September 1965

[3] R. Gaylor and A. Et, "An adaptive tracking filter for bendng mode stabilization," Journal of Space Crafts and Rockets, vol. 4. pp. 573-577, 1967.

[4] W. B. Wie and K. W. Byun, "New generalized structural filtering concept for active vibration control synthesis," Journal of Guidance Control and Dynamics, vol. 12, pp.147-154, 1989.

[5] A. Elmelhi, M. Yasir, and X. Jiang, "Structural filters for stabilizing a flexible launch vehicle," presented at the International Conference on Electrical Engineering, July 10-14, 2005, Kumming, China.

[6] H. Hosenthien, T. Michael and T. Borelli, Phase Adaptive Stabilization of Bending for Large Aerospace. Vehicle Boosters, Astronic Reaseach and Development Rept., No. 2, NASA-TM X-53044, May 1, 1964.

[7] R. Gaylor, S. Robert and C. David, "Adaptive tracking filter for bending mode stabilization," J. SPACECRAFT. vol. 4, pp. 573-577, May 1967

[8] R. L. Hardy, "Multiquadric equations of topography and other irregular surfaces," Journal of Geophysical Research. vol. 76, pp.1905-1915, 1971.

[9] D. Broomhead and D. Lowe, "Multivariable functional interpolation and adaptive networks," Complex Systems, vol. 2, pp. 321-355, 1988.

[10] A. V. Saunchez, Neurocomputing-Special Issue on RBF Networks, Part I, 1998.

[11] S. Haykin, Neural Network-A Comprehensive Foundation, Second Edition, Prentice-Hall, 1999.

[12] G. Fasshauer, Mesh Free Approximation Methods with MATLAB, Singapore: World Scientific, 2007.

[13] L. Zhou, F. Shumin and J. Changsheng, "Adaptive integral dynamic surface control based on fully tuned radial basis function neural network," Journal of Systems Engineering and Electronics, vol. 21, pp. 1072-1078, 2010.

[14] L. Greensite, Control Theory: Volume II, Analysis and Design of Space Vehicle Flight Control Systems, Spartan Books, 1970.

[15] A. Elmelhi, "Flight vehicle's stabilization based fuzzy logic control design and optimization," Ph. D Thesis, Beijing University of Aeronautics and Astronautics, Beijing, China, Nov.2005.

[16] C. Cunningham and L. Schaeperkoetter, Study of Applications of A Tracking Filter to Stabilize Large Flexible Launch Vehicle, Phase I, Final Technical Report Procurement Request, May 1966.

[17] L. Cheng, G. Zhang, B. Wan, L. Hao, Q. Hongzhi and D. Ming, "Radial basis function neural network-based PID model for functional electrical stimulation system control," in Proc. 31st Annual International Conference of the IEEE EMBS, Minneapolis, USA, 2009, pp.3481-3484.

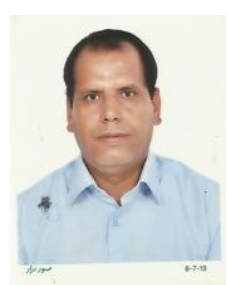

Ali Elmelhi was born in Tripoli, Libya, received B .Sc degree from Tajora engineering AcademyLibya in the field of Communication Engineering in 1989, the M Sc degree from Technical collage -Cairo-Egypt in the field of Electrical engineering in 1995 and $\mathrm{Ph}$. D. Degree in the field of Navigation, Guidance and Control from Beijing University of Astronautical and Aeronautical- Beijing (BUAA) China in 2005.From 1990 to 2003 he was a research engineer in the research center -Tripoli Libya, and the main interested area during this time period is in flight control design and modeling. From 2004 he starts to work as a lecturer in the university of Tripoli -Libya. The previous publications are mainly in the area of fuzzy logic and neural network applications for control design. In addition, the stabilization of the launch vehicles based on the optimization techniques such as Genetic algorithm and optimal control are the another parts for his publications field. The current interest area is in control system design and optimization based on Linear Matrix In-equality.

Dr Ali Elmelhi is an Associate Prof and a member of IEEE. 